\title{
sur la gestion et les finances publiques En Juin 2021
}

\section{BUDGET DE L'ÉTAT ET DES OPÉRATEURS}

\section{Données générales sur les finances publiques}

Le Gouverneur de la Banque de France affiche son optimisme

Dans 3 interviews données à la presse (Radio Classique, Europe 1, Le Figaro), François Villeroy de Galhau, Gouverneur de la Banque de France, est revenu sur l'état de l'économie française.

Pour le Gouverneur, I'économie française accélère sur la route de la sortie de crise. Cela est visible notamment dans le secteur des services, en premier lieu l'hôtellerie-restauration. Globalement, après la chute de 2020, la croissance française atteindrait plus de 5,75 \% en 2021, soit davantage que la moyenne européenne à $4,6 \%$, et mieux que l'Allemagne et l'Italie. L'activité devrait retrouver son niveau d'avant-crise un peu plus tôt que prévu, dès le début 2022. La consommation des ménages et l'investissement des entreprises sont les deux principaux moteurs de ce rebond.

Concernant l'endettement, le Gouverneur a précisé que l'annulation de la dette publique française, y compris celle détenue par la banque centrale, était une illusion. Pour François Villeroy de Galhau le désendettement se fera grâce à trois leviers, dont chacun est nécessaire: du temps, de la croissance, et une meilleure efficacité des dépenses publiques.

Une stratégie de finances publiques pour la sortie de crise

En février dernier, le Premier ministre a confié à la Cour des comptes une mission sur la stratégie de finances publiques d'après-crise. Le 15 juin 2021, la Cour répond à cette saisine en publiant un rapport dans lequel elle conclut notamment que la crise sanitaire laissera des marques durables sur l'économie française et les finances publiques. Dans ce contexte, la Cour considère que la soutenabilité de la dette publique deviendra un enjeu de souveraineté. Pour les magistrats de la rue Cambon, il s'agit d'une condition nécessaire pour faire face aux chocs économiques futurs et rester en mesure de financer les politiques et le fonctionnement des administrations publiques.

Pour assurer cette soutenabilité, la Cour propose une stratégie reposant sur deux piliers : le renforcement de la croissance potentielle et la maîtrise des dépenses publiques. Cet effort devra s'accompagner d'une rénovation du cadre de la gouvernance des finances publiques, d'une amélioration de la qualité de la dépense publique dans cinq secteurs prioritaires (retraites, assurance maladie, politique de l'emploi, minima sociaux, politique du logement) et du renforcement de l'équité et de l'efficience des politiques et des administrations publiques.

À cette fin, la Cour formule 27 recommandations à destination de l'exécutif.

\section{$\lambda$ Procédure budgétaire}

Rapport sur les crédits ouverts par décret d'avance

En application de l'article $58-6^{\circ}$ de la loi organique relative aux lois de finances (LOLF), le 18 juin 2021, la Cour des comptes publie un rapport sur les crédits du budget de l'État ouverts par décret d'avance, suite à la saisine du ministre de l'économie, des finances et de la relance et du ministre délégué chargé des comptes publics du 6 mai 2021.

Le décret d'avance du 19 mai 2021 qui avait disparu depuis 2018 a fait un retour remarqué 
dans le contexte particulier de la crise sanitaire. Les restrictions d'activité décidées au premier trimestre 2021 ont conduit le Gouvernement à prolonger plusieurs dispositifs d'aide aux ménages et aux entreprises. Les crédits disponibles en 2021 pour le financement de ces dispositifs ne suffisaient pas pour couvrir les consommations de l'année, compte tenu de la prolongation des aides. Dans l'attente du vote de la loi de finances rectificative, le projet de décret d'avance a organisé un redéploiement de crédits vers ces aides.

Dans son rapport, la Cour des comptes estime que les conditions de procédure du décret d'avance ont été respectées : respect du plafond, équilibre des ouvertures et annulations, situation d'urgence.

La Cour ne manque toutefois pas de souligner que dans son rapport sur le budget de l'État en 2020, elle a estimé qu'il aurait été possible de ne pas reporter les crédits excédentaires pour l'exercice 2020 et que les crédits nécessaires en 2021 auraient dû être ouverts et justifiés en loi de finances initiale, ce qui aurait permis d'éviter une certaine confusion des exercices, contraire au principe d'annualité budgétaire, et d'affecter la portée de l'autorisation parlementaire.

Enfin, la Cour observe que l'annulation d'une partie des crédits du programme 356 (activité partielle) non consommés en 2020 pour les reporter en 2021 sur le programme 357 (fonds de solidarité), pourtant réalisée tardivement (le 18 mars 2021), a porté sur un montant excessif (2,3 Md€). Le décret d'avance a en effet dû ouvrir des crédits sur le programme 356 pour le financement de l'activité partielle en 2021. Dans son analyse de l'exécution budgétaire en 2020, la Cour s'était d'ailleurs étonnée de l'ampleur du report au bénéfice du fonds de solidarité au regard des besoins prévisibles en 2021 pour l'activité partielle.

\section{Administrations budgétaires}

\section{Bilan du rattachement de la gendarmerie au ministère de l'intérieur}

À la demande de la commission des finances du Sénat, la Cour des comptes a réalisé un bilan - publié le 30 juin 2021 - du rattachement organique et fonctionnel de la gendarmerie nationale au ministère de l'intérieur, intervenu en 2009. À cette occasion, le législateur avait souhaité garantir le statut militaire des gendarmes, tout en précisant leurs missions. Les pouvoirs publics disposent aujourd'hui de deux forces distinctes et complémentaires, avec la police en milieu urbain et la gendarmerie en milieu périurbain et rural.

La Cour relève que la répartition territoriale entre les deux forces doit cependant mieux tenir compte des évolutions des populations et de la délinquance. L'exercice par les deux forces de la mission de police judiciaire relève de gestions encore trop séparées, notamment en matière de criminalité organisée, de cybercriminalité, ou d'emploi des forces d'intervention. Les pratiques dans ces différents domaines devraient être rapprochées et rendues plus efficientes. Aussi, le rapprochement des services de police technique et scientifique est souhaitable et reste à réaliser. Pour la Cour, la mutualisation de formations continues communes pourrait être développée au-delà de l'existant.

D'un point de vue budgétaire, le principe de «parité globale » a permis aux gendarmes de bénéficier des évolutions statutaires et indemnitaires obtenues par les policiers, ce qui a contribué à augmenter la masse salariale de la gendarmerie de $22 \%$ en 10 ans. Cette situation grève les marges de manœuvre budgétaires nécessaires aux investissements et aux équipements des deux forces.

La Cour estime que la pérennisation d'un effort budgétaire important au cours des prochaines années est nécessaire pour maintenir la capacité opérationnelle de la gendarmerie. En matière immobilière, faute d'investissement suffisant pendant de nombreuses années, la gendarmerie est confrontée à des besoins de réhabilitation importants, dont le montant est estimé à $300 \mathrm{M} €$ par an, alors que les crédits consommés sur la période 2009 à 2009 représentaient moins de $90 \mathrm{M} €$ en moyenne annuelle. En 2021, l'investissement immobilier devrait s'élever à $140 \mathrm{M} €$ en crédits de paiement contre $118 \mathrm{M} €$ en loi de finances initiale pour 2020. Ces montants demeurent cependant inférieurs aux besoins identifiés par la gendarmerie pour rénover son parc immobilier. Par ailleurs, cet effort financier sera ponctuel et ne réglera pas le passif cumulé depuis de nombreuses années, que seule une programmation financière pluriannuelle réaliste et soutenable pourrait traiter.

La Cour, qui a déjà appelé l'attention sur la situation des forces de sécurité à cet égard, estime nécessaire de freiner l'inflation des recrutements et des mesures indemnitaires afin de dégager des marges de manœuvre pour les dépenses d'investissement et de fonctionnement. 


\section{Budget des pouvoirs publics}

Certification des comptes 2020 de l'Assemblée nationale et du Sénat

Le 2 juin 2021, la Cour des comptes a publié ses rapports de certification des comptes 2020 de l'Assemblée nationale et du Sénat.

La Cour certifie que les comptes de l'Assemblée nationale et du Sénat pour l'exercice clos le 31 décembre 2020 sont, dans leurs aspects significatifs, réguliers et sincères, et qu'ils donnent une image fidèle de leur situation financière et de leur patrimoine.

Sans remettre en cause son opinion générale, la Cour a néanmoins appelé l'attention sur quatre notes de l'annexe aux états financiers concernant les comptes de l'Assemblée nationale et sur 3 notes concernant les comptes du Sénat.

\section{FinANCES LOCALES}

\section{$\lambda$ Rapport de la Cour des comptes sur les finances publiques locales - Fascicule $n^{\circ} 1$}

Le premier enseignement de cette nouvelle " cuvée » de la Cour des comptes concerne le suivi de la crise sanitaire et financière sur le plan local. Les magistrats estiment qu'à ce jour, l'impact de la crise est moins élevé que les premières estimations, avec une épargne globale en baisse de $4 \mathrm{Md}$. Cela s'explique par des recettes fiscales, pour l'instant, non impactées comme la CVAE mais également par des charges de fonctionnement maîtrisées avec des hausses très modérées. Bien évidemment, la baisse de l'investissement explique aussi cette situation. Pour la Cour, la baisse de 7,1 \% en année électorale est similaire à celle de 2014, ce qui toutefois mérite une analyse plus poussée car le niveau d'investissement sur le cycle actuel demeure beaucoup plus bas que le précédent. Un autre grand mérite de ce rapport est de revenir sur la hausse de l'endettement des régions.

\section{$\pi$ Guide pratique de la taxe de séjour}

Ce nouveau guide édité par la DGCL permet de revenir sur les nouveautés introduites par la loi de finances pour 2021. A partir de 2021, l'article 123 de la loi de finances pour 2021 prévoit une seule date limite de délibération, à savoir le $1^{\text {er }}$ juillet pour une application à compter du

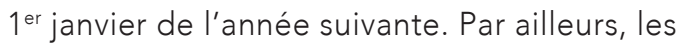
hébergements non classés ou en attente de classement seront taxés dans la limite du tarif le plus élevé adopté par la collectivité (article 124 de la loi de finances pour 2021). Notons également que lorsqu'une collectivité territoriale décide d'appliquer le régime forfaitaire pour certains hébergements touristiques, elle peut adopter un abattement facultatif applicable au nombre d'unités de capacité d'accueil de l'établissement en fonction de la durée de la période d'ouverture.

\section{$\lambda$ Loi de finances rectificative}

Le Gouvernement a déposé 8 juin 2021 un nouveau projet de loi de finances rectificative afin d'ajuster les données financières avec les réalités économiques. En matière de droit des collectivités territoriales, l'un des objectifs consiste à indemniser toujours au mieux les collectivités territoriales. L'article 10 instaure une compensation des pertes de recettes tarifaires subies par les SPIC en régie et instaure un fonds d'urgence au titre des pertes de recettes tarifaires des SPA exploités par le bloc communal à cause de l'épidémie de covid-19, calculée en fonction de l'évolution de l'épargne brute entre 2019 et 2020. Cet article crée également un fonds d'urgence pour les communes et leurs groupements exploitant un service public administratif (SPA) en régie, et dont l'équilibre financier a été fragilisé par une dégradation significative des recettes du SPA.

\section{Rapport de la délégation aux collectivités territoriales sur les métropoles}

Dans la perspective du débat parlementaire concernant le projet de loi 4D, la délégation du Sénat a souhaité rendre un rapport pour discuter de l'avenir des métropoles. La première recommandation en matière financière concerne le fait que le contrat de plan État-région comprenne un volet sur la coopération métropoles-territoires voisins. Ce rapport s'étonne également qu'il n'y ait aucune particularité en matière de subventions pour les métropoles. L'une des contreparties qui est proposée concerne la DGF avec l'introduction d'une bonification, sans que cela porte atteinte aux communes. La prochaine loi de finances pourrait aborder cette piste.

\section{$\lambda$ Informations diverses sur la DGF}

La dotation globale de fonctionnement est en constante évolution. À la suite de l'échec de la réforme de 2015, la part dédiée à l'intercommunalité a été réformée. Mais cela n'est pas 
suffisant et de nombreux points demeurent en suspens comme le rappelle l'audition du directeur général des collectivités locales par la Délégation aux collectivités territoriales et à la décentralisation. Ainsi en est-il du financement des parts de péréquation ou encore de la question du financement des communes peu peuplées. Cette audition permet également d'apporter de premiers éléments sur la réforme des indicateurs, avec sans doute l'intégration des droits de mutation à titre onéreux (DMTO) dans le calcul des indicateurs financiers. On peut enfin relever la volonté de la DGF de faciliter la connaissance de cette dotation avec la mise en place d'une cartographie interactive et d'un guide.

\section{$\lambda$ Pré-Rapport de l'Observatoire des finances et de la gestion publique locales Les finances des collectivités locales en 2021}

La DGCL vient de rendre son traditionnel rapport en décidant de publier ce qu'elle appelle un «pré-rapport». Ce document revient longuement sur les conséquences financières de la crise. En premier lieu, I'Observatoire rappelle que pour la première fois depuis cinq ans le besoin de financement est de - 0,5 Md€ avec cependant de grandes inégalités entre catégories de collectivités territoriales (régions et collectivités territoriales uniques (CTU) sont en besoin de financement de - 2,3 MdE alors que le bloc communal présente une capacité de financement de $+2,0 \mathrm{Md}$.

En toute logique, I'encours de dette augmente de $+3,3 \%$. Toutefois, cette hausse de l'encours de dette ne correspond pas à un accroissement des dépenses d'investissement mais à une réduction importante de celles-ci de - 6,2\%. En parallèle de cela, la hausse de l'encours correspond à une forte dégradation de l'épargne brute de $-10,8 \%$ et un allongement du délai de désendettement de $+0,7$ an.

\section{$\lambda$ Des directeurs de projet et experts de haut niveau dans les collectivités territoriales}

Un projet de décret fixe les dispositions relatives aux emplois d'expert de haut niveau et de directeur de projet pouvant être créés dans les collectivités territoriales et leurs établissements publics. Il définit les emplois concernés, prévoit les modalités de sélection des candidats et les conditions d'emploi.
Selon le texte, les experts de haut niveau et les directeurs de projets peuvent être " chargés d'animer la conduite de projets et de coordonner à cette fin l'action des services intéressés ou d'assurer des missions de conseil, d'audit ou de médiation qui requièrent une expérience diversifiée et une grande capacité d'analyse et de proposition. Les missions confiées peuvent évoluer pendant la durée d'occupation des fonctions » (article 2). Par ailleurs, les « experts de haut niveau et les directeurs de projet sont placés auprès de l'autorité territoriale ou, sur sa décision, auprès du directeur général des services de la collectivité ou de l'établissement public assimilé » (article 3).

\section{FINANCES SOCIALES}

\section{$\pi$ Situation des comptes sociaux et conséquences de la crise sanitaire}

Le premier projet de loi de finances rectificative pour 2021 assure le financement des mesures d'urgence jusqu'à l'été et met en place le dispositif de soutien aux entreprises pour la sortie de crise

Les ministres de l'économie et des finances et des comptes publics ont présenté au Conseil des ministres du 2 juin le premier PLFR pour 2021 ; ce projet doit permettre de valider les mesures (à hauteur de 7,2 Md€) du décret d'avances du 19 mai (voir les Repères de mai) ; il complète ces mesures (3,6 MdE pour le Fonds de solidarité et 2,2 Md€ pour l'activité partielle auxquels s'ajoutent $4 \mathrm{Md} €$ pour compenser les exonérations de cotisations sociales, de façon à assurer le service de ces aides jusqu'à la fin de l'été), de même qu'il prévoit 4,2 Md€ au titre de la mise en place du Plan de relance. II introduit par ailleurs de nouveaux dispositifs pour préparer les entreprises à la sortie de crise : $2 \mathrm{Md€}$ pour mener des opérations de participation financières de l'État qui ne sont pas liées directement aux effets de la crise ; 0,6 Md€ pour le «Fonds de transition pour les entreprises", pour apporter un concours en fonds propres aux entreprises dont la solvabilité est dégradée. Le total des ouvertures de crédit au titre des mesures de soutien s'élève donc à 16,6 Md€. Le PLFR procède à des ouvertures de crédits (qui sont gagées) dans le domaine social : 0,7 Md€ pour l'hébergement d'urgence, 0,13 Md€ pour le «Pass'Sport », 0,15 Md€ pour le financement des bourses étudiantes. Une des mesures les plus commentées a été, par ailleurs, I'assou- 
plissement du " carry-back » (suppression du plafonnement) pour apporter un soutien en trésorerie des entreprises.

La stratégie de finances publiques pour la sortie de crise : la Cour des Comptes met la pression sur la réduction de la dépense sociale

En réponse au lancement par le Premier ministre, en février dernier, d'une mission sur la stratégie de finances publiques d'après crise, la Cour des Comptes a rendu son rapport le 15 juin. Partant du constat d'une situation des finances publiques françaises déjà bien détériorées avant la crise, la Cour estime que celle-ci a fait perdre, en 2021, 2 points de capacité de production par rapport à la trajectoire d'avantcrise. Après que les années 2021 et 2022 auront été consacrées au « débranchement « progressif des mesures de soutien au revenu des ménages et des entreprises, la Cour préconise, à partir de 2023, la mise en œuvre de réformes de gouvernance et de méthode des finances publiques, et, sur le fond, concentre l'effort de maîtrise de la dépense quasi exclusivement sur 5 politiques sociales: les retraites, le système de santé, l'indemnisation du chômage et les aides à l'emploi, les minima sociaux et la politique du logement) s'agissant des retraites, la Cour se prononce pour une réforme paramétrique, avec une préférence implicite pour le relèvement de l'âge légal ; pour les dépenses de santé, elle préconise la stabilisation de leur part dans le PIB, qui passe par une bien meilleure coordination de l'offre de soins entre les 3 secteurs (hôpital, ville et médico-social); en ce qui concerne l'assurance chômage et la politique de l'emploi, la recommandation principale de la Cour vise à donner au fonctionnement du régime un caractère beaucoup plus contra-cyclique, en mettant la pression sur le retour à l'emploi et l'allongement de la durée des contrats lorsque la conjoncture s'améliore ; s'agissant des minima sociaux, elle relève que la dérive des dépenses résulte à la fois de la faiblesse des taux de sortie vers l'emploi et de l'articulation peu cohérente des différents types de prestations; enfin elle recommande une réforme en profondeur de la politique du logement, à la fois très coûteuse en France et peu efficace, en simplifiant les dispositifs, et en concentrant les aides sur les publics les plus défavorisés.

La Commission des comptes de la Sécurité sociale : le déficit 2021 plus élevé que prévu
Le rapport de la Commission des comptes de la Sécurité sociale publié le 24 juin rend compte des exercices 2020 et 2021. S'agissant des comptes 2020, le déficit du Régime général et du FSV est arrêté à -38,7 MdE, après intégration d'un concours exceptionnel du Fonds de Réserve des retraites de $5 \mathrm{Md} \epsilon_{\text {, ce qui est }}$ moins que ce qui était prévu dans la dernière LFSS (-49 d€), du fait d'une contraction de la masse salariale moindre que redouté $(-5,7 \%$ contre $-8,9 \%$ prévus); toutes les branches sont déficitaires en 2020, les déficits les plus importants étant concentrés sur l'Assurance maladie $(-30,4 \mathrm{Md})$ et l'ensemble Vieillesse +FSV $(-6,2 M d €)$. Pour 2021, le déficit du Régime général et du FSV serait de -38,4 Md€, en aggravation de -2,6 Md€ par rapport à la prévision de la LFSS pour 2021. Si les recettes sont meilleures qu'attendu $(+6,5 \mathrm{Md})$, sauf pour les cotisations des travailleurs indépendants, les dépenses de santé dérapent de 9,1Md€ par rapport à l'objectif de l'ONDAM, du fait des surcoûts induits par la $3^{e}$ vague de la COVID19; au total, le coût des dépenses exceptionnelles de santé liées à la crise sanitaire aura été de 13,4 Md€ en 2021, dont 4,6 Md€ pour la vaccination et 4,9 Md€ pour les tests. Et le dérapage de I'ONDAM par rapport à la situation d'avant crise de 2019 aura été de 16,4 \% à périmètre constant. Par branche, le déficit serait de - 31 Md€ pour la Maladie, de - 4,4 Md€ pour la Vieillesse, de -1,2 Md€ pour l'autonomie et de - 2,6 Md€ pour le FSV; seraient en excédent les branches Accident du travail (+ 0,3 Md€) et Famille (+ 0,5 Md€). La situation de trésorerie de I'ACOSS, qui avait atteint son point bas à $-89,7$ Md€ le 22 juin 2020 et - 50,6 Md€ le 31 décembre 2020 va être améliorée par le transfert progressif, sur plusieurs années, de $136 \mathrm{Md}$ à la CADES prévu par les lois organique et ordinaire du 7 août 2020 (voir Repères d'août 2020) : 20 Md€ ont été ainsi transférés en 2020, suivis de 40 Md€ en 2021. Parallèlement, la Caisse des Dépôts a repris sa place dans les financeurs de premier rang de l'ACOSS, puisque la part des instruments de marché est passée de $94 \%$ en 2019 à $76 \%$ en 2020.

\section{$\lambda$ Assurance maladie/Hôpital}

\section{Un plan de $7 \mathrm{Md} €$ pour les industries de santé}

En Conseil stratégique des industries de santé, le Président de la République a présenté le 29 juin la stratégie innovation santé pour 2030. Cette stratégie vise à faire de la France la première nation européenne innovante et souve- 
raine en matière de santé, après qu'on a rendu le sous-investissement dans ces industries depuis une trentaine d'années responsable de l'impréparation de la France face à la crise de la COVID 19. Elle prévoit, sur plusieurs années, une enveloppe de $7 \mathrm{Md}$ : $1 \mathrm{Md}$ € pour renforcer notre capacité de recherche biomédicale ; 2,2 Md€ pour investir dans les domaines de santé de demain (biothérapies, médecine 5P - préventive, personnalisée, prédictive, participative et basée sur les preuves, préparation aux pandémies) ; 1,5 Md€ pour pérenniser la relocalisation en France des industries de la santé ; 2 Md€ pour renforcer I'investissement de BPIFrance dans le domaine de la santé. Par ailleurs, une Agence d'innovation en santé sera créée.

\section{$\pi$ Retraites}

La remise du rapport Turquois-Causse sur les petites retraites

Les députés Nicolas Turquois et Lionel Causse ont remis au Premier ministre leur rapport sur les petites retraites. Fixant à $1000 €$ le seuil d'une "petite pension ", les rapporteurs constatent qu'environ $1 / 3$ des retraités se situent en deçà de ce seuil de revenu, avec une surreprésentation des femmes, ainsi que des non-salariés et des exploitants agricoles. Les causes sont imputées à la complexité des régimes et des minima de pensions, bien que les prestations non contributives (Allocation de solidarité aux personnes âgées-ASPA- qui vient compléter les faibles revenus jusqu'à $903 €$ ) réduisent effectivement les inégalités; cela aboutit à ce que $24 \%$ des assurés ne liquident pas I'ensemble de leurs droits à retraite. Les auteurs du rapport comptent plus sur les mécanismes assurantiels que sur les minimas sociaux pour redresser la situation des petites retraites. Audelà de l'amélioration de l'information sur les droits à retraite, ils préconisent notamment de revaloriser à $85 \%$ du SMIC les pensions des retraités actuels ayant atteint le taux plein et validé 120 semestres. Le coût de cette revalorisation serait de 2,1 Md€ en 2025. Selon les rapporteurs, il pourrait être financé par une réduction de l'abattement de $10 \%$ à I'IR sur les pensions et retraites, ou par l'alignement du taux normal de CSG des retraités sur celui des actifs (avec, en contrepartie la suppression de la cotisation maladie de $1 \%$ sur la retraite complémentaire), ou par la création d'une deuxième journée de solidarité, voire l'augmentation des cotisations pour la retraite.

\section{La réforme des retraites de nouveau en question}

En visite dans le Lot le 3 juin, le Président de la République a créé la surprise en annonçant pour l'été « des décisions difficiles » qui pourraient « ne pas rendre possible une nouvelle candidature ». Derrière ces propos sibyllins, tous les commentateurs ont alors pensé à une reprise de la réforme des retraites, pour laquelle le Président a lui-même concédé qu'elle ne pourrait pas être la même que le projet de réforme systémique de I'hiver 2019-22020. Parmi les pistes "paramétriques » envisagées : une intensification de la "réforme Touraine «sur la durée de cotisation, le parachèvement de la réforme des régimes spéciaux, le passage de l'âge légal de départ de 62 à 64 ans. Cette dernière option paraissait "tenir la corde» après les élections régionales, des annonces pouvant intervenir dès la première quinzaine de juillet.

\section{Le Conseil d'orientation des retraites re- voit un peu à la baisse les prévisions de déficit des régimes de retraite}

Le Conseil d'orientation des retraites a rendu le 10 juin son rapport annuel sur l'évolution des systèmes de retraite, à moyen terme (2030) et à long terme (2070). Selon les scénarios habituels d'évolution du PIB (entre $1,0 \%$ et 1,8\%) qui ont été rappelés dans les Repères de novembre, l'évolution tendancielle fait apparaître que la part des dépenses de retraite dans le PIB partirait d'un niveau exceptionnellement élevé en $2020(14,7 \%$, du fait de la très forte contraction du PIB) pour s'établir à 13,7\% du PIB en 2030 (ce qui est moins que dans les prévisions du COR de novembre dernier) et dans une fourchette comprise entre $11,3 \%$ et $13 \%$-selon les scénarios de croissance- en 2070 (ce qui est inférieur de près d'1/2 point de PIB par rapport aux prévisions de l'an dernier). La baisse tendancielle du poids des dépenses de retraite dans la richesse nationale est donc confirmée. Pour déterminer le besoin de financement global du système de retraite, le COR reprend ses conventions comptables habituelles relatives à l'effort de l'État pour financer les retraites de la fonction publique et des régimes spéciaux, qu'il croise avec les 4 hypothèses de croissance. Il en ressort qu'après avoir connu un besoin de financement exceptionnel de 0,8 \% du PIB en 2020, le solde de l'ensemble des régimes obligatoires de base serait compris dans une fourchette de - 0,1\% à - 0,7 \% du PIB en 2030 et une fourchette de $+2,1 \%$ à $-0,7 \%$ du PIB en 2070 ; le retour à l'équilibre serait assuré en 
2060 dans 3 scénarios de croissance sur 4, ce qui est plus favorable que dans les prévisions de novembre dernier. Cette inflexion paraît due à une moindre croissance des dépenses (pour une part effet de la surmortalité due à la Covid en 2020), et à une révision à la hausse des prévisions du PIB.

Les partenaires sociaux se penchent sur le déficit de I'AGIRC-ARRCO

Selon les informations parues dans Les Échos, syndicats et patronat ont ouvert le 21 juin des discussions en vue du redressement du régime de retraite complémentaire des salariés du privé. Le régime, qui avait dégagé un excédent de près d'1 Md€ en 2019, a accusé en 2020 un déficit de 4,1 Md€ (voir Repères de mars). Suite à l'accord de 2019, les gestionnaires se sont fixé comme règle de conduite d'avoir en réserve au moins 6 mois de prestations, ce qui, suivant les hypothèses démographiques, pourrait ne plus être assuré à partir de 2029. Les pistes restent ouvertes entre un gel des pensions ou une augmentation des cotisations.

Le rapport Blanchard-Tirole propose une version améliorée du système universel de retraites par points

Le rapport commandé par le Président de la République à un groupe d'économistes piloté par Olivier Blanchard et Jean Tirole préconise, entre de nombreuses propositions, de mettre en place un système universel de retraite par points. Reprenant le diagnostic qui avait soustendu le projet du gouvernement adopté par l'Assemblée nationale en mars 2020, et en panne depuis cette date, le rapport suggère toutefois de très nombreuses modifications visant notamment à rendre plus transparentes la fixation et l'évolution de la valeur du point (modalités dont la complexité dans le projet gouvernemental avaient fini par en ruiner la crédibilité), et en confiant la mise en œuvre à un organisme indépendant. En récusant l'idée d'un âge légal unique et universel de départ à taux plein, les économistes préfèrent plutôt une « fenêtre de départ ", avec un mécanisme de surcote et de décote pour décaler l'âge moyen de départ vers 64 ans. Pour accentuer la redistributivité du système, ils proposent l'acquisition de points gratuits par les travailleurs aux salaires les plus faibles. Enfin, ils recommandent de compléter le mécanisme d'équilibrage financier du futur régime par un levier démographique, en de dégradation du rapport cotisants/pensionnés.

\section{Assurance chômage}

L'UNEDIC annonce des perspectives financières moins dégradées pour l'Assurance chômage, mais elles sont aussitôt compromises par la décision du Conseil d'État sur la réforme du régime

Le bureau de I'UNEDIC a présenté le 17 juin des perspectives financières pour le régime d'Assurance chômage moins dégradées que celles de février dernier (voir Repères de février). Pour 2021, le déficit serait de - $12 \mathrm{Md€}$ (- 6,5 Md€ hors coût de l'activité partielle) et une quasi atteinte de l'équilibre serait possible en 2023. Ces prévisions se fondent sur I'hypothèse d'un retour à l'activité d'avant crise dès le début de 2022 et d'une baisse du chômage indemnisé de - 154000 allocataires à fin 2021, mais elles prennent en compte une entrée en vigueur complète des nouvelles dispositions sur l'indemnisation, alors que l'application de celles-ci, notamment le calcul du salaire journalier de référence, viennent d'être suspendues par le Conseil d'État le 22 juin, lequel ne se prononce cependant pas sur le fond. En cas de non application de la réforme, le déficit serait dégradé dans une fourchette supplémentaire entre - $1 \mathrm{Md} €$ et - 2,8 Md€. En ce qui concerne la dette, elle serait de 69 Md€ à fin 2022 (vs 70,6 Md€ dans la prévision de février), mais replongerait à 74 Md€ en 2023 en cas d'annulation complète de la réforme.

\section{$\lambda$ Formation professionnelle}

\section{Le déficit du financement de la formation professionnelle s'aggrave}

Selon des informations parues dans Les Échos, le besoin de financement du dispositif de formation professionnelle, qui avait déjà été évoqué dans les Repères de décembre 2020, va en s'aggravant, puisque France Compétences pourrait accuser un déficit de - 2,5 Md€. Cela est dû au succès de la réforme de la formation professionnelle de 2018, notamment à l'envolée du compte de formation professionnelle (CFP), mais également aux mesures en faveur de l'emploi des jeunes et des chômeurs, mises en œuvre pour lutter contre la crise, qui ont largement reposé sur la formation : apprentissage, primes à l'embauche, plan d'investissement dans les compétences, projets de transition professionnelle...La ministre du travail va prochainement recevoir les partenaires sociaux, et, en s'inspirant en partie des propositions du rapport IGF-IGAS d'avril 2020, revoir à la baisse le coût de prise en charge de certaines formations. 


\section{\ Solidarité/lutte contre la pauvreté/ Revenu universel}

Le rapport 2021 de l'INSEE sur les revenus et patrimoines des ménages

Le traditionnel rapport de l'INSEE sur les revenus et patrimoines de ménages prend en compte cette année une approche plus large de la redistribution, en intégrant une valorisation monétaire de l'effet des transferts par les services publics. Selon l'INSEE, I'écart de revenu primaire entre les $10 \%$ les plus modestes et les $10 \%$ les plus aisés est de 1 à 13 ; il est ramené de 1 à 7 par les transferts monétaires comme les prestations et les prélèvements, et le serait de 1 à 3 en prenant en compte les transferts en nature comme l'éducation, la santé et le logement qui contribueraient à hauteur de $50 \%$ à la réduction des inégalités.

\section{$\lambda$ Contrôles/lutte contre la fraude}

La lutte contre la fraude : baisse des redressements dans la lutte contre le travail dissimulé

Le bilan de la lutte contre la fraude en matière de travail dissimulé, publié par l'ACOSS le 16 juin a fait apparaître une baisse de $15 \%$ (605,7 M€) des cotisations redressées par rapport à 2019, ce qui s'explique par le fait que les inspecteurs des URSSAF ont dû limiter leurs investigations sur le terrain et que les difficultés de nombreuses entreprises ont nécessité une adaptation des contrôles. La lutte contre la fraude au détachement demeure une priorité, avec, en 2020, une attention particulière portée au contrôle de l'activité partielle, dans le cadre d'échanges d'informations croisées avec les corps de contrôle du ministère du travail. En ce qui concerne la fraude aux prestations, la branche Famille a enregistré également une baisse des redressements (255 M€ vs $323 \mathrm{M} €$ en 2019), tout en renforçant sa traque des "fraudes à enjeu » qui mettent en œuvre des mécanismes sophistiqués de détournement des allocations (ex. RSA, APL, allocations familiales)

\section{FISCALITÉ ET PROCÉDURES FISCALES}

\section{$\pi$ Politique fiscale}

La participation de la France à la lutte européenne contre les pratiques fiscales abusives

L'évasion fiscale et la planification fiscale agressive des grandes sociétés multinationales pri- veraient les États de I'UE de plus de 50 Mds€ de recettes par an. Ces pratiques sont facilitées par l'absence de toute obligation, pour les grandes sociétés multinationales, d'indiquer, État par État, où elles réalisent leurs bénéfices et où elles paient leurs impôts dans I'UE. Le texte sur lequel un accord est intervenu impose aux entreprises multinationales ou aux entreprises autonomes, qu'elles aient ou non leur siège dans I'UE, dont le chiffre d'affaires total consolidé dépassait $750 \mathrm{M} €$ pour chacun des deux derniers exercices financiers consécutifs, de publier des informations relatives à l'impôt sur les bénéfices dans chaque État membre, ainsi que dans chacun des États tiers énumérés à l'annexe I des conclusions du Conseil relatives à la liste de l'UE des États et territoires non coopératifs à des fins fiscales ou énumérés depuis deux années consécutives à l'annexe II de ces conclusions du Conseil. Cette publication peut avoir lieu au moyen d'un modèle commun au niveau de I'UE. La directive comporte une liste complète et définitive des informations qui doivent être divulguées. La déclaration sera faite dans un délai de douze mois à compter de la date de clôture du bilan de l'exercice financier en question. La directive fixe les conditions dans lesquelles une société peut obtenir le report de la divulgation pour une période maximale de cinq ans. Elle détermine également à qui incombe effectivement la responsabilité de veiller au respect de l'obligation de déclaration. Les États membres disposeront de dix-huit mois pour transposer la directive dans leur législation nationale. Quatre ans après la date de transposition de la directive, la Commission présentera un rapport sur l'application de cette dernière. Le texte sur lequel un accord provisoire est intervenu va maintenant être soumis aux instances compétentes du Conseil et du Parlement européen. Attendons désormais la décision que le Conseil constitutionnel rendra au sujet de la loi de transposition de cette directive. Elle sera très certainement instructive ! Le législateur français avait tenté, dès décembre 2016, d'introduire unilatéralement ce dispositif, à l'article 137 de la loi relative à la transparence, à la lutte contre la corruption et à la modernisation de la vie économique, qui avait été censuré par le Conseil constitutionnel (Cons. const., 8 déc. 2016, décis. $n^{\circ}$ 2016-741 DC, §100 à §104) au nom de la liberté d'entreprendre bien que le législateur ait entendu, par une mesure de transparence, éviter la délocalisation des bases taxables afin de lutter contre la fraude et l'évasion fiscales, en poursuivant 
ainsi un objectif de valeur constitutionnelle. À suivre...

\section{$\lambda$ Impôt sur le capital}

Les recommandations fiscales du rapport Blanchard-Tirole (1)

La commission installée par le Président de la République en mai 2020 sur " Les grands défis économiques ", sous l'égide des économistes Olivier Blanchard et Jean Tirole, avec I'appui de France Stratégie, vient de rendre son rapport. Concernant les inégalités, la commission propose des aménagements de la fiscalité française.

En premier point, la commission Blanchard-Tirole constate que les possibilités d'échapper à l'impôt sur les successions sont assez nombreuses dans le système actuel (exonération de l'assurance-vie, exonération Dutreil sur la transmission des entreprises familiales, etc.). Le rapport propose de restructurer l'imposition des transmissions pour qu'elle soit fondée sur le bénéficiaire et qu'elle soit progressive en fonction du montant cumulé reçu par ce dernier. Au lieu d'imposer les transmissions à chaque décès, le nouveau système imposerait la totalité des transmissions (donations, héritages, toutes sources confondues) dont l'héritier a bénéficié, de sorte que ceux qui reçoivent davantage soient imposés à des taux plus élevés. Des taux préférentiels et réduits fondés sur la relation entre le donateur et l'héritier resteraient possibles. En outre, il serait possible de tenir compte de l'âge du bénéficiaire à la date de la transmission. Cet impôt devrait avoir une assiette très large, incluant la plupart ou la totalité des actifs, en limitant les types d'actifs n'entrant pas dans la base imposable et les exonérations. L'abattement sur la totalité des donations pourrait être relativement élevé.

En deuxième point, le rapport préconise de travailler sur la question de la taxation optimale des plus-values mobilières en privilégiant, comme dans certains États, la taxation du stock de capital et non le flux de celui-ci, ce qui pose la question de la taxation des plus-values latentes. À ce jour, la législation interdit d'enregistrer les plus-values latentes, en vertu du principe de prudence. La plus-value latente est un simple espoir de bénéfice qui se cristallise ou non lorsque le bien sort du bilan de l'entreprise. Or, la comptabilité peut uniquement prendre en compte les bénéfices effectivement réalisés par l'entreprise et non les espoirs de gain. Tant que le bien ne sort pas du bilan, il ne sera pas soumis à imposition. Les plus-values latentes ne sont donc pas taxées à ce jour. S'agissant de l'exonération de plus-value immobilière au titre de la résidence principale, le rapport préconise qu'elle ne s'applique «qu'aux propriétés d'une valeur inférieure à un certain seuil, qui pourrait être fixé à un niveau suffisamment élevé pour que les classes moyennes puissent continuer à en bénéficier largement ". S'agissant des réductions d'impôt au titre de la loi Pinel pour les investissements dans certains logements neufs, le rapport préconise de mener des évaluations très rigoureuses afin de déterminer si elles permettent effectivement d'atteindre les objectifs visés par le législateur. Plus généralement, le rapport propose de développer une réflexion critique assortie d'analyses coûts/avantages avant toute entrée en vigueur de nouvelles exemptions fiscales et dispositions spécifiques.

\section{$\pi$ Contrôle fiscal}

\section{Les recommandations fiscales du rapport Blanchard-Tirole (2)}

Pour lutter contre la fraude fiscale, le rapport Blanchard-Tirole préconise d'exploiter de manière plus systématique les possibilités offertes par le Big Data et l'analyse de données pour détecter les fraudes et tracer les contribuables. Il propose de réorganiser l'infrastructure technologique de l'administration fiscale et de développer des outils avancés d'analyse en investissant dans les technologies numériques appropriées (logiciel et matériel).

\section{$\lambda$ Impôt sur les sociétés}

\section{Décret n²021-705 du 2 juin 2021 et ré- duction du taux de TASCOM}

La taxe sur les surfaces commerciales (TASCOM) s'applique aux établissements de vente au détail d'une surface de plus de $400 \mathrm{~m}^{2}$, mais également aux établissements de moins de $400 \mathrm{~m}^{2}$ contrôlés directement ou indirectement par une même personne (réseau intégré) et exploités sous une même enseigne commerciale lorsque la surface totale des points de vente excède $4000 \mathrm{~m}^{2}$. En application des dispositions législatives (L. $n^{\circ} 72-657$ du 13 juillet 1972 art. 3 al. 18) en faveur de certaines catégories de commerçants et artisans âgés, le taux de la TASCOM fait l'objet d'une réduction, fixée par décret à $20 \%$, pour les seuls établissements dont la surface est comprise entre $400 \mathrm{~m}^{2}$ et $600 \mathrm{~m}^{2}$, lorsque leur chiffre d'affaires par mètre carré n'excède pas $3800 €$. Le législateur 
(L. n²020-1721 du 29 déc. 2020 de finances pour 2021, art. 136) a modifié cette disposition afin de faire également bénéficier de la réduction de taux les établissements assujettis à la TASCOM dont la surface de vente au détail est inférieure à $400 \mathrm{~m}^{2}$. Désormais, la réduction de taux de 20 \% s'applique donc, sous les mêmes conditions de chiffre d'affaires par mètre carré, à tous les établissements dont la surface des locaux de vente destinés à la vente au détail est inférieure à $600 \mathrm{~m}^{2}$. Ce décret du Premier ministre (JORF nº128 du 4 juin 2021) fixe les modalités et le taux de cette réduction, et procède également à plusieurs mises à jour en supprimant des dispositions obsolètes du texte.

Accord sur de nouvelles règles internationales concernant la fiscalité des entreprises

Lors du sommet du G7 le 5 juin 2021, les ministres des finances des États-Unis, du Royaume-Uni, du Canada, de I'Allemagne, de la France, de l'Italie et du Japon se sont engagés sur de nouvelles règles mondiales concernant la fiscalité des entreprises, reposant sur un taux d'imposition de $15 \%$ des plus grandes multinationales telles que Google ou Apple. Ce projet d'accord doit recueillir l'approbation du G20 en juillet 2021. Selon certains observateurs, (v. not. F. Douet, Fiscalité 2.0 - Fiscalité du numérique, LexisNexis, coll. "Précis fiscal», 3e éd., 2020), un taux minimum de $15 \%$ ne veut pas dire grandchose, tout dépend de l'assiette. Par ailleurs, I'accord obtenu au G7 concernant la meilleure répartition des recettes fiscales entre États pourrait avoir des conséquences inattendues puisque l'entreprise Amazon pourrait y échapper, alors que les entreprises françaises Air Liquide, LVMH ou L'Oréal en remplissent bien les critères... Par ailleurs, cet accord va avoir des conséquences majeures sur la comptabilité des grandes entreprises. Le Conseil d'analyse économique (CAE), sous l'égide du Premier ministre, a chiffré l'impact de l'imposition minimum des profits des multinationales à l'étranger. Avec un taux de $15 \%$, les gains pour la France s'élèveraient à $6 \mathrm{Mds} € /$ an, puis $2 \mathrm{Mds} € /$ an si les "paradis fiscaux» relèvent leurs taux. Et ils seraient proportionnellement plus élevés que ceux de l'Allemagne et des États-Unis. Finalement si cette imposition voit le jour, ce qui n'est pas encore évident au niveau des États membres de l'OCDE, elle devrait rapporter un peu plus de 4 Mds€/an à la France.

\section{$\pi$ Impôt sur le revenu}

\section{Commentaire au BOFIP de la convention fiscale franco-monégasque}

La convention fiscale bilatérale du 18 mai 1963 et la convention tendant à éviter les doubles impositions et à codifier les règles d'assistance en matière successorale du $1^{\text {er }}$ avril 1950 entre la France et la Principauté de Monaco ont fait I'objet de commentaires au BOFIP-impôts le 2 juin 2021 afin d'actualiser les précédents commentaires de 2012 sur les règles d'imposition des revenus des personnes physiques et morales et les règles d'imposition en matière successorale résultant de ces deux conventions.

Le critère de la nationalité pour déterminer la résidence fiscale en France

Selon l'article $4 \S 1$ de la convention fiscale bilatérale du 9 septembre 1966 entre la France et la Suisse, afin d'éviter les doubles impositions en matière d'impôt sur le revenu et sur la fortune, une personne est considérée comme « résident d'un État contractant » lorsque, en vertu de la législation dudit État, elle se trouve assujettie à l'impôt dans cet État, à raison de son domicile, de sa résidence, de son statut juridique, de son siège de direction ou de tout autre critère de nature analogue. Dans un arrêt récent (CE, 9 juin 2021, 9e ch., M. A. et Mme D., Req. $n^{\circ}$ 431551), le Conseil d'État a précisé que le principe en la matière est d'examiner successivement et dans l'ordre de la convention les différents critères aboutissant à la définition du terme de résident et non pas d'examiner ces critères de manière alternative. A l'issue de son raisonnement, le Conseil d'État a considéré qu'il n'était pas possible de déterminer l'État où $M$. A. séjournait de façon habituelle. La CAA, en jugeant que M. A. ayant la nationalité française pour les années litigieuses, et qu'à ce titre, il devait être regardé comme domicilié fiscalement en France, n'a pas commis d'erreur de droit selon le Conseil d'État.

\section{Une proposition de loi visant à créer une réduction d'impôt sur le revenu pour les bénévoles associatifs}

Plus de 150000 associations ont recours à de l'emploi salarié en France. À elles seules, elles emploient 1,8 millions de salariés sur le territoire national (3,5\% du PIB). Afin d'inciter de nouvelles personnes à s'investir dans le bénévolat afin de relancer la vie associative, cette proposition de loi déposée à l'Assemblée nationale (Doc. parl. $n^{\circ} 4249,15$ juin 2021) visant à créer une réduction d'impôt sur le revenu 
pour les bénévoles qui s'investissent dans une association. Cette réduction d'impôt sur le revenu serait par ailleurs octroyée sur le même principe que l'abandon des frais au profit des associations, qui est déjà en vigueur pour les bénévoles qui engagent des dépenses dans le cadre de leur activité associative. Ce dispositif a fait ses preuves et très peu d'abus ont été constatés (CGI, art. 200-1).

\section{$\pi$ Fiscalité écologique}

La fin de l'avantage fiscal du gazole non routier : 2022 ou 2023 ?

Après un report de la suppression du GNR, annoncé au 1er Janvier 2023 par le Gouvernement le 25 mai dernier (cf. Repères de mai 2021), une initiative, non concertée à l'Assemblée nationale, le 11 juin 2021, de certains députés du groupe majoritaire a avancé ce report au 1 er juillet 2022, réduisant de six mois le délai de 18 mois décidé par le Gouvernement !

\section{Management public}

\section{$\lambda$ Politique de modernisation}

Le bilan du plan de transformation de I'ENA

Le 16 juin 2021, le conseil d'administration de I'ENA a été l'occasion de présenter le bilan du plan de transformation de l'école initié au mois de novembre 2018. On y retrouve les avancées des trois dernières années suivant 5 orientations majeures :

- Favoriser la diversité : des profils variés dans tous les recrutements;

- Favoriser la proximité : des élèves au plus près des réalités de terrain et des fonctionnaires au plus près des enjeux actuels de l'action publique ;

- Ouvrir, décloisonner: des partenariats avec le monde universitaire et les autres écoles de service public ;

- Rayonner à l'international : influence française à l'étranger ;

- Améliorer la gestion : redressement financier, réorganisation des moyens humains et éco- responsabilité.

En termes de recrutement, il est également fait mention de l'existence de nouveaux concours, notamment un recrutement au profit des titulaires d'un doctorat ainsi que l'ouverture, en 2021, du concours externe «Talents » réservé aux boursiers.

\section{$\lambda$ Relations avec les usagers}

\section{Le rapport d'activité 2020 du Conseil d'État}

Le Conseil d'État vient de publier son Rapport public - Activité juridictionnelle et consultative des juridictions administratives en 2020. Cette étude est consacrée, en particulier, aux conséquences de la crise sanitaire sur son fonctionnement. Ainsi, dès le début du confinement, l'activation du plan de continuité d'activité de la haute juridiction a eu pour but de permettre la poursuite de ses missions essentielles de justice administrative tout en assurant la sécurité des personnels et des publics.

Rétrospectivement, la mise en place de l'état d'urgence sanitaire s'est traduite par une production normative importante concernant tous les domaines de l'action publique : gestion des urgences sanitaires, mesures de soutien aux entreprises, temps de travail, chômage partiel, organisation des élections, aides diverses...

Par ailleurs, comparativement à l'année 2019, le contentieux de cassation ( $59 \%$ du total des entrées contentieuses) a connu une diminution de près de $30 \%$ des pourvois présentés contre des décisions des juridictions administratives spécialisées (894 pourvois en 2020 contre 1265 en 2019).

Enfin, marquée par la pandémie de Covid-19, l'année judiciaire a vu le nombre de demandes de protection baisser de $22 \%$ devant la Cour nationale du droit d'asile (CNDA). En 2020, la Cour a enregistré 46043 affaires, contre 59091 en 2019.

\section{$\lambda$ Gestion publique}

L'accompagnement du secteur public par des cabinets privés

Le Cercle de la Réforme de l'État a publié le 21 juin 2021 une note relative au recours à l'expertise externe par les administrations. Le texte relève que l'utilisation par les organisations publiques d'experts externes n'est pas une nouveauté : elle " est largement ancrée depuis plusieurs dizaines années dans les pratiques managériales ». Cependant, " des interrogations et des craintes quant à un usage massif et incontrôlé de ces expertises par l'État ont récemment été soulevées notamment par des parlementaires à l'occasion de la gestion de la crise Covid».

Il est incontestable que le recours à l'expertise externe a été dans certains cas un moyen de compenser le manque de ressources humaines 
et/ou de compétences pénalisant les organisations concernées dans la mise en œuvre de leurs projets de transformation, voire dans l'exécution même de leurs missions. Cependant, si le recours à l'apport externe dans les organisations publiques peut être nécessaire et utile «pour accompagner les évolutions des organisations publiques, il reste qu'une prestation intellectuelle quelle qu'en soit la nature ne s'achète pas comme une "commodité". Le processus d'achat et de pilotage de ce type de prestations requiert une forte implication $d u$ commanditaire et une "maîtrise d'ouvrage" de la prestation forte et structurée».

\section{L'arrêt « Direction générale de la cohésion sociale " de la CDBF}

La Cour de discipline budgétaire et financière (CDBF) a condamné dans un arrêt du 14 juin 2021 I'ancienne directrice générale de la cohésion sociale (DGCS) à $500 €$ d'amende pour avoir attribué un marché en 2013 alors même qu'elle "ne disposait pas de tous les éléments juridiquement requis pour apprécier les capacités techniques, professionnelles et financières " du fournisseur. En l'espèce, il s'agissait d'un marché d'expérimentation d'une prestation de centre relais téléphonique pour les personnes sourdes ou malentendantes.

\section{Immobilier public}

La direction de l'immobilier de l'État a publié le 22 juin 2021 son rapport L'immobilier public de demain. Ce document souligne que la crise sanitaire a modifié le rapport que les agents ont avec leur lieu de travail, dans le contexte d'une banalisation du télétravail. Il énonce également que l'apparition de nouvelles formes d'organisation des espaces dans le secteur privé « est venue questionner la pertinence des modèles immobiliers en place dans la sphère publique ».

Enfin, face à un parc immobilier ancien et souffrant d'un manque d'attractivité en termes d'utilisation et de services rendus à ses utilisateurs, "ces évolutions des modes de travail font émerger une aspiration à un renouveau de l'immobilier public, plus moderne, adaptable et à même d'accueillir des pratiques plus collaboratives $"$.

\section{EUROPE}

\section{Pacte de stabilité}

Paquet "Semestre européen " du printemps : ouvrir la voie à une reprise forte et durable
Le 2 juin, la Commission européenne a présenté le paquet de printemps du Semestre européen, qui met l'accent sur la fourniture d'orientations budgétaires aux États membres, engagés dans un processus de réouverture progressive de leur économie. Ces orientations visent à aider les États membres à consolider la reprise économique en utilisant au mieux la facilité pour la reprise et la résilience (FRR), instrument clé au cœur de NextGenerationEU. Le Semestre européen a été adapté cette année, compte tenu des liens avec les plans pour la reprise et la résilience des États membres, et il définit les investissements et les réformes qui seront financés par le FRR.

\section{$\lambda$ Budget européen}

État de droit : les députés pressent la Commission à défendre les fonds européens

Le mercredi 2 juin, les députés ont demandé à la Commission européenne de prouver qu'elle est capable de défendre le budget de I'UE contre les États membres violant le principe de l'État de droit. Durant la session plénière du mois, les députés débattront de l'application des règles adoptées en 2020 sur la conditionnalité liée à l'État de droit pour l'accès aux fonds de l'UE.

\section{Fiscalité}

L'Union européenne trouve un accord politique pour renforcer la transparence fiscale des multinationales

Le 2 juin, L'Union européenne trouve un accord politique pour renforcer la transparence fiscale des multinationales. L'accord vise notamment à obliger les grands groupes à déclarer leurs bénéfices dans chacun des pays de I'Union européenne où ils opèrent. Les eurodéputés et le Conseil européen, qui représente les États membres de l'Union européenne (UE), ont trouvé mardi $1^{\text {er }}$ juin un accord politique sur une nouvelle directive pour renforcer la transparence fiscale des multinationales. Cet accord prévoit que les grands groupes dont le chiffre d'affaires annuel est supérieur à $750 \mathrm{M€}$ seront obligés de déclarer leurs bénéfices, leur nombre d'employés et le montant de leurs impôts dans chacun des pays de I'UE où ils opèrent, ainsi que dans les juridictions figurant sur la liste noire des paradis fiscaux de I'Union.

L'obligation de «reporting public pays par pays» a été saluée par plusieurs groupes politiques au Parlement européen, dont les sociaux-dé- 
mocrates et les Verts, comme un progrès important pour plus de justice fiscale. Mais de nombreuses ONG et la gauche radicale ont émis des critiques, jugeant cette proposition « inopérante ».

L'accord sur une nouvelle directive, issue d'une proposition de la Commission européenne présentée en 2016, doit encore être formellement approuvé par les eurodéputés en séance plénière et par le Conseil européen. II intervient au moment où des discussions à l'Organisation de coopération et de développement économiques (OCDE) sur la taxation des bénéfices des multinationales ont été relancées par une proposition du président américain, Joe Biden, $d^{\prime}$ introduire un taux minimum d'imposition de $15 \%$.

\section{$\lambda$ États-membres}

Le Conseil adopte une recommandation adressée à la Roumanie pour déficits excessifs

Le 18 juin, Le Conseil a adopté une recommandation au titre de la procédure concernant les déficits excessifs à l'égard de la Roumanie. La recommandation prévoit que la Roumanie devra mettre fin à la situation de déficit excessif en 2024 au plus tard. En avril 2020, le Conseil avait adopté une recommandation à la Roumanie en vue de mettre un terme au déficit excessif en 2022 au plus tard mais, en raison de l'incertitude causée par la pandémie de Covid-19, la Commission n'a pas présenté de recommandation à la Roumanie en novembre 2020.

\section{INTERNATIONAL}

\section{$\pi$ Monnaies}

La Fed maintien ses taux mais anticipe l'inflation

Lors de la réunion de son comité de politique monétaire du 16 juin 2021, la Réserve fédérale américaine a laissé ses taux d'intérêts inchangés, entre 0 et $0,25 \%$. Mais elle a annoncé qu'une hausse interviendrait d'ici à la fin de 2023, soit avec un an d'avance par rapport au calendrier initial, en raison des tensions inflationnistes. Néanmoins, les marchés financiers se sont rapidement apaisés.

Aurélien BAUDU (Fiscalité et procédure fiscale), Fabrice BIN (Europe, international),

Florent GAULLIER-CAMUS (budget de l'État et des opérateurs),

Matthieu HOUSER (finances locales), Christophe SINNASSAMY (management public)

Yves TERRASSE (finances sociales), Robin DEGRON (coordination).

Retrouvez sur www.rgfp.fr :

- I'intégralité des REPÈRES d'actualité des finances et de la gestion publiques depuis 2015 - le 15 du mois, les REPÈRES d'actualité du mois précédent. 